\title{
Judith A. Sugar, Robert J. Riekse, Henry Holstege and Michael A. Faber (eds.) (2014). Introduction to Aging. A Positive, Interdisciplinary Approach. New York: Springer, 317 pp. ISBN 9780826108807 (paperback)
}

\author{
REVIEWEd by SHYH POH TEO*
}

Gerontology is a broad and interdisciplinary subject, covering aspects of psychology, humanities, policy, economics, education, law and medicine. It is no easy feat to represent these multiple perspectives in a concise publication; yet, these authors managed to do so in a simplified manner that is appropriate for an introductory gerontology textbook.

The consistent layout of the book facilitates assimilation of knowledge in gerontology, and provides a good balance between theory and practical information. For those new to gerontology, it provides a useful foundation of principles that can be applied to practice. Chapters start with learning objectives, sometimes supplemented with scenarios and thought-provoking questions on real-life issues that older people face. At the end of each unit is a "Practical Application" section, offering reflective comments on how and why these topics are socially relevant. Interspersed throughout the book are links to video clips offering stories and narratives, illustrating how the concepts described are utilised.

The book covers a very wide range of topics, reflecting the interdisciplinary nature of gerontology as a subject. General trends regarding older

* Shyh Poh Teo, Geriatrics and Palliative Unit, Department of Internal Medicine, RIPAS Hospital, Brunei Darussalam 
International Journal of Ageing and Later Life

people are described in terms of physical changes, living conditions, retirement and medical care systems. The historical perspectives and social theories presented in the book make fascinating reading. It is interesting to read about the evolution of how we perceive older people and how public policies change over time after deliberate consideration of issues ranging from income, health care, housing, transportation and long-term care.

One highlight of reading this book is the refreshingly positive attitude expressed by the authors regarding ageing. The stereotypical assumptions about older people as being in poor physical and mental health or as living in poverty are challenged with information about older people living happy, active and productive lives. There is a dedicated section in the book about maintaining wellness, providing details of proper nutrition, physical activity and sleep hygiene. Common medical conditions in old age are discussed, with preventative recommendations provided to reduce risk of developing disease, such as appropriate vaccinations and screening. Participation in society is encouraged, and generic advice is given to overcome barriers, for example, difficulties in obtaining or maintaining employment.

There are several themes covered in the book which deserve specific mention, and which I certainly appreciate being included in this book. One of these is the chapter on sexuality. Intimacy and closeness are integral aspects of life and a basic emotional need which continues to old age, yet it is rarely discussed openly with older people. Death, dying and bereavement are described as well, with a section on how to cope from loss of loved ones. Practical tips are provided on caring for a family caregiver and how to deal with resistance of accepting care. The chapter covering elder abuse and scams affecting vulnerable older people included real examples of court cases. This was eye opening and motivates better public awareness about these problems.

The main downside is that some parts of the book are quite specific to the US, which limits generalisability, particularly to developing countries. Specifically, significant detail is provided on Social Security, Medicare, legislation public policies and services available. However, these aspects are still useful to read as they are described in an accessible manner and valuable lessons could also be extrapolated for a comparative perspective. For example, formal support systems such as home-based services, adult 
day care, nutrition services and case management are generic approaches which even developing countries could adopt if useful.

Readers should also be aware there is significant diversity between older people and there will be exceptions to the facts presented in the book. For example, older people were sub-classified into the young-old (65-74 years), aged (75-84 years) and the oldest-old (85 years and older), and general descriptive characteristics of each category were given. The assumption that older people all fit nicely into these compartments risks ageism, overlooking the individuality of each person, with unique preferences and wishes.

Overall, this book successfully pulls together diverse concepts and allows readers to see the bigger picture, which is essential to enable a coordinated effort from multiple disciplines when considering the challenges and opportunities of a rapidly ageing population. The book is true to its title, sharing a positive, interdisciplinary approach on ageing with its readers. It is also a very thought-provoking text, being upfront in posing questions or ethical dilemmas to the readers. For advocates of older people, this would be an excellent text to have as an introductory book to navigate the dynamic field of gerontology and as a bridge to further learning. 